\title{
Chronic cardiac toxicity after inhalation of 1,1,1-trichloroethane
}

\author{
ANDREW A MCLEOD, ROBERT MARJOT, MARK J MONAGHAN, PHILIP HUGH-JONES, \\ GRAHAM JACKSON
}

Abstract
Two patients showed evidence of chronic cardiac toxicity after
repeated exposure to 1,1,1-trichloroethane. In both cases there
was circumstantial evidence of a deterioration after routine
anaesthetic use of the related compound halothane. An ado-
lescent boy who sniffed trichloroethane presented with multiple
ventricular arrhythmias during tonsillectomy. Follow up showed
mild chronic left ventricular impairment. A 54 year old man had
repeated industrial exposure to trichloroethane and deteriorated
from mild stable cardiac failure to end stage cardiac failure after
halothane anaesthesia for herniorhaphy. Chronic cardiac
toxicity is a previously unreported feature of this type of solvent
exposure.
Related compounds such as halothane may have a toxic interaction after exposure to trichloroethane.

\section{Introduction}

Inhalational abuse of organic solvents ("glue sniffing") is common among young adolescents. Organic solvents are also widely used in industry, where occupational exposure may be a hazard. Many reports have focused on sudden death, which is often believed to be mediated by the known enhanced sensitivity to endogenous catecholamines. ' Chronic cardiac effects have not been extensively documented. Some solvents have a close chemical similarity to volatile anaesthetic drugs, particularly the halogenated hydrocarbons, and the concept of possible adverse interaction has not been recognised. We report two cases in which repeated exposure to

King's College and Dulwich Hospitals, London SE5 9RS

ANDREW A MCLEOD, MD, MRCP, consultant cardiologist

King's College Hospital, London SE5 9RS

ROBERT MARJOT, MB, DA, registrar in anaesthesia

MARK J MONAGHAN, MSC, senior chief technician

PHILIP HUGH-JONES, MD, FRCP, honorary consultant physician

King's College and Lewisham Hospitals, London SE5 9RS

GRAHAM JACKSON, FRCP, consultant cardiologist

Correspondence to: $\mathrm{Dr}$ A A McLeod, Cardiac Department, King's College Hospital, London SE5 9RS. 1,1,1-trichloroethane produced serious cardiac toxicity. Anaesthesia with halothane was induced in both patients for non-related conditions and was followed by a deterioration in cardiac rhythm or performance.

\section{Case histories}

CASE 1

A. 14 year old boy was well before tonsillectomy but was noted to have multiple ventricular extrasystoles after the start of anaesthesia. Premedication was with papaveretum $10 \mathrm{mg}$ and hyoscine hydrobromide $0.2 \mathrm{mg}$ intramuscularly. Induction was with thiopentone $200 \mathrm{mg}$ followed by gallamine $10 \mathrm{mg}$, and paralysis was achieved with suxamethonium $75 \mathrm{mg}$. The patient was maintained with nitrous oxide, oxygen, and halothane. Multiple ventricular extrasystoles and runs of ventricular tachycardia were noted. His blood pressure remained normal and arterial gas analysis showed good oxygenation and a normal acid-base state. The halothane was changed to enflurane and subsequently isoflurane without any effect on the arrhythmias. They were reduced in complexity and frequency, however, by two $50 \mathrm{mg}$ boluses of intravenous lignocaine. Postoperative recovery was normal, but complex arrhythmias continued, responding moderately to further lignocaine and then to disopyramide $450 \mathrm{mg}$ by mouth daily. A Bruce protocol treadmill test to assess antiarrhythmic efficacy was performed to 13 minutes. Immediately afterwards, however, 15 seconds of nodal bradycardia ( 30 beats $/ \mathrm{min}$ ) followed by six seconds of asystole occurred. A multiprogrammable demand pacemaker was inserted. Despite increasing the disopyramide to $600 \mathrm{mg}$ a day isolated ventricular extrasystoles, couplets, and ventricular tachycardia recurred. Oral mexiletine $600 \mathrm{mg}$ daily was substituted and he completed a further treadmill test without any arrhythmias. A subsequent 24 hour electrocardiogram showed no ventricular tachycardia, but around 4000 ventricular extrasystoles occurred.

Some form of drug or solvent abuse was suspected but strenuously denied. Results of clinical cardiac examination remained normal. An echocardiogram showed normal cardiac anatomy but mild left ventricular dilatation and impaired contraction (fig 1). The boy was reviewed as an outpatient. Complex ventricular arrhythmias recurred and continued. He complained of dizzy spells and blackouts. A 24 hour electrocardiogram showed recurrent ventricular tachycardia. Treatment with amiodarone 200 $\mathrm{mg}$ twice daily was begun with almost immediate abolition of symptoms, and mexiletine was withdrawn after one week. Subsequently his mother discovered that a one litre bottle of trichloroethane kept in the shed by her husband for professional cleaning of audiovisual equipment was almost empty. A large amount of Tipp-Ex typewriter correcting fluid thinner (also containing trichloroethane) had also disappeared.

Three months after starting amiodarone, and almost six months after 
initial presentation, the patient was asymptomatic. His electrocardiogram showed frequent single ventricular extrasystoles but no other abnormality. The echocardiogram was unchanged; left ventricular end diastolic dimension was $5 \cdot 9,5 \cdot 5$, and $5.4 \mathrm{~cm}$ at presentation, two months, and five months, respectively (95\% prediction limits for the patient 3.9 and $4.4 \mathrm{~cm}){ }^{2}$ Fractional shortening (percentage change of left ventricular dimension during systole) at the same times was $20 \%, 20 \%$, and $15 \%$, respectively $(95 \%$ prediction limits $28 \%$ and $44 \%$ ). ${ }^{2}$ Home sources of solvent abuse were not available and he denied further sniffing, though he may still have been doing so.

\section{CASE 2}

A 54 year old man began welding in 1974 . He worked five hours a day with mild and stainless steel, which was degreased by Genklene (trichloroethane). $\mathrm{He}$ also worked close to baths containing trichloroethane. Between 1974 and 1979 he suffered a series of illnesses diagnosed as bronchitis, sinusitis, or influenza. He gradually became more breathless so that he had to rest in bed for several days. He was at that time the only person consistently exposed to the grease solvents. In March 1979 much more trichloroethane was being used on rough metal. Far more fumes than usual were generated and he became acutely ill. He was, admitted to hospital in atrial fibrillation and congestive heart failure. He was stabilised with medical treatment but did not return to his previous occupation.

In 1982 he was referred for cardiac assessment in relation to industrial injury compensation. He was free of symptoms, taking digoxin $0.25 \mathrm{mg}$ daily. He denied alcohol excess and did not smoke cigarettes. Echocardiography showed a slightly dilated left atrium and poor left ventricular function compatible with a congestive cardiomyopathy. Cardiac catheterisa-
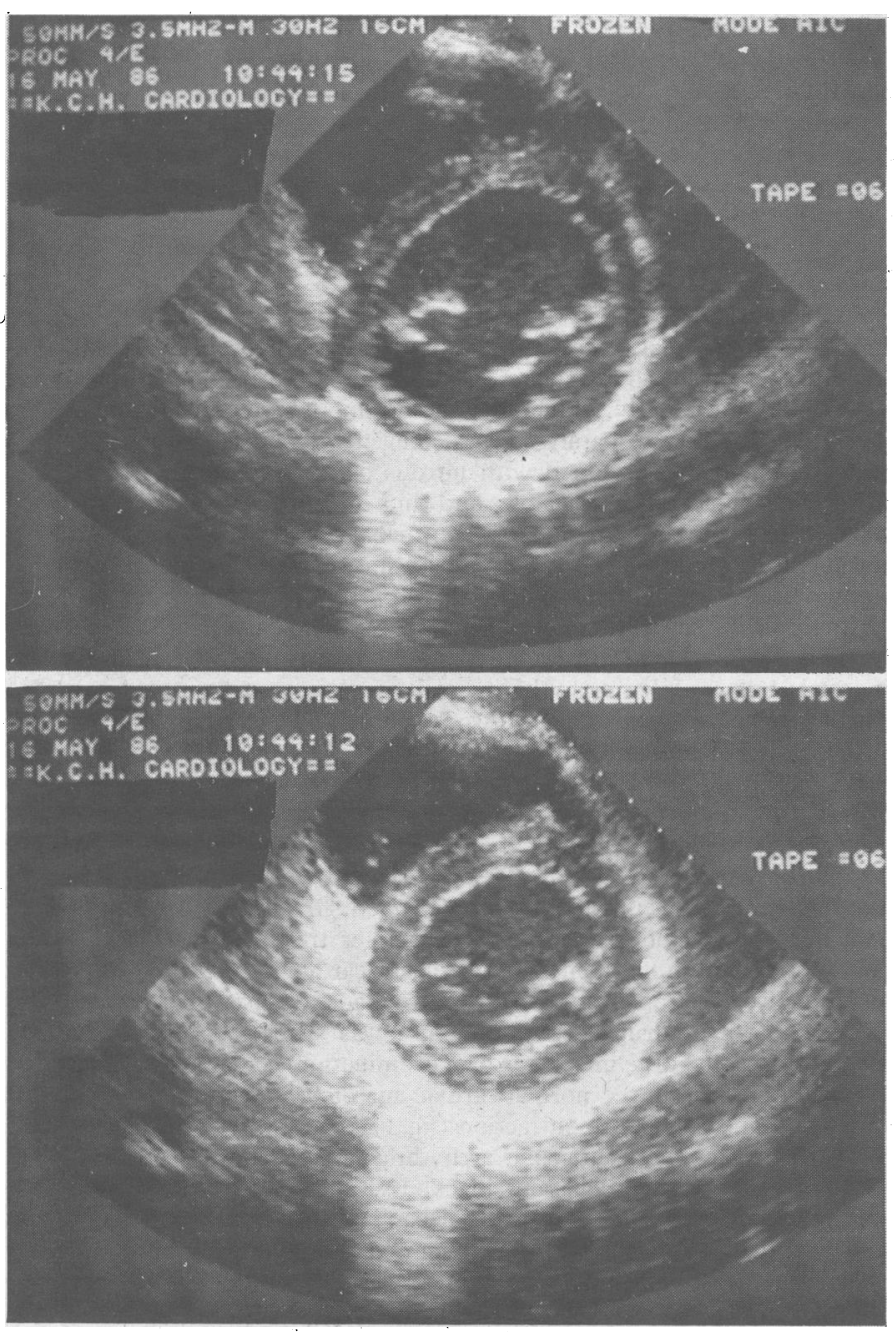

FIG 1-Case 1. Cross sectional echocardiograms two days after presentation. Short axis view of left ventricle at level of mitral chordae. Top: End diastole. Bottom: End systole. Dimensions were $5.5 \mathrm{~cm}$ and $4.3 \mathrm{~cm}$, respectively. Fractional shortening was $22 \%$. Neither frame is of postextrasystolic beat.
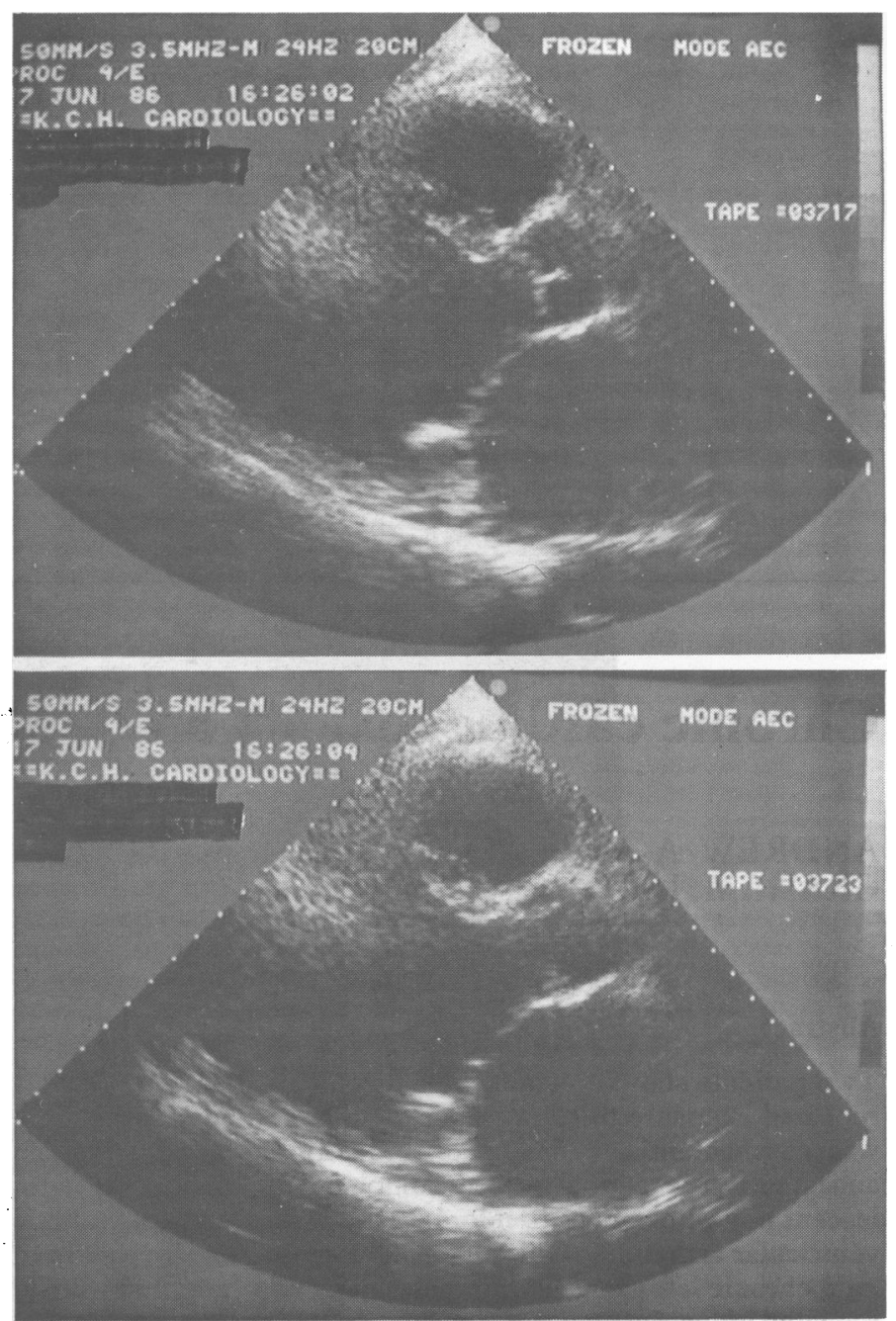

FIG 2-Case 2. Long axis echocardiograms showing (top) end diastolic and (bottom) end systolic images of left ventricle. Cavity is dilated and shows little change in chamber size after systolic contraction.

tion disclosed normal coronary arteries, an apical cardiomyopathy, and normal pulmonary artery pressure. There was mild mitral regurgitation. He was anticoagulated. He continued stable and well, working in his own business, until September 1985, when he was given a general anaesthetic for repair of an inguinal hernia. Routine halothane anaesthesia was used. Subsequently he rapidly became considerably more breathless but was not reinvestigated until February 1986.

There had been a substantial deterioration in left ventricular function. Comparison with 1982 showed an increase in left atrial size from $4 \cdot 3$ to $7 \cdot 0$ $\mathrm{cm}$, and the left ventricular dimensions had increased from 5.4 to $7.3 \mathrm{~cm}$ at end diastole and from 4.2 to $6.5 \mathrm{~cm}$ at end systole (fig 2). The patient was admitted to hospital in congestive cardiac failure. Stabilisation on this occasion required digoxin, frusemide, and captopril. Cardiac catheterisation and angiography confirmed the deterioration in cardiac function. A series of left ventricular biopsies was performed and the samples analysed at the National Heart Hospital, London. Histological examination showed a severely hypertrophied myocardium with evidence of dilatation and changes compatible with a healing or healed myocarditis. No virus particles were found on electron microscopy. Without knowledge of the time course of the illness the report suggested that a recent episode, perhaps six months previously, had exacerbated the underlying problem.

The patient was stabilised with medical treatment but in June 1986 he was again admitted in severe heart failure. The echocardiogram was unchanged. A right heart catheter excluded pulmonary hypertension. Ambulatory electrocardiography over $\mathbf{2 4}$ hours showed, in addition to atrial fibrillation, frequent episodes of ventricular tachycardia. The serum potassium concentration was normal, as was the digoxin concentration. Amiodarone abolished the ventricular arrhythmias and digoxin was stopped as a precautionary measure because of the potential for interaction. Again the patient was stabilised, but in September he was readmitted in cardiac failure. When last seen he remained severely limited and was being assessed for cardiac transplantation. 


\section{Discussion}

The Health and Safety Executive has recently reviewed the toxicity of 1,1,1-trichloroethane in man and animals. ${ }^{1}$ The report highlights the acute cardiac toxicity of the compound found during trials as an inhalational anaesthetic in the 1950s. Hypotension and ventricular arrhythmias were observed. More recently several sudden deaths after solvent abuse or due to industrial accidents were attributed to a combination of factors such as narcosis, suffocation or hypercapnia (when rebreathing from a plastic bag), or cardiac arrhythmias possibly associated with hypercapnia or with exercise. ${ }^{13}$ Central nervous system damage with impairment of central control of respiration has also been suggested. ${ }^{4}$ In a single case cardiac arrhythmias were noted to persist for up to two weeks after exposure, ${ }^{5}$ but in that instance very high concentrations of 1,1,1-trichloroethane were present and urine assay showed continuing excretion of 1,1,1-trichloroethane and 1,1,2-trichloroethane during the period of observation. Chronic cardiac toxicity has not been reported.

Our two cases show that chronic myocardial damage presenting either as ventricular arrhythmias or as left ventricular failure is a possible sequel to both long term industrial exposure and short term solvent abuse with trichloroethane. These cases are important and also unusual in that exposure to trichloroethane was comparatively "pure." This is uncommon among glue sniffers, who may abuse multiple compounds.

Of main concern is the possible provocation of symptoms or rapid deterioration by a volatile anaesthetic. Halothane (2-bromo-2chloro-1,1,1,-trifluoroethane) may produce cardiac arrhythmias by sensitisation to catecholamines; nevertheless, it is less toxic in this regard than other halogenated alkanes, ${ }^{6}$ though more so than the newer ethers such as isoflurane and enflurane. The circumstances in which our first patient presented and the second patient deteriorated suggest that preliminary damage was caused by trichloroethane. Possibly a subsequent cross reaction with halothane may have occurred. Endomyocardial biopsy rarely gives a specific diagnosis, as many pathological insults may result in a largely similar histological picture. It is striking, however, that the pathologist, without knowing the second patient's clinical history, suggested a subacute myocarditis superimposed on a picture compatible with longstanding damage.

Trichloroethane is a widely available solvent and degreasing agent, being found in various glues, typewriter correction fluid, dry cleaning aids, and plaster remover. Its acute toxicity has made it unsuitable as a volatile anaesthetic and its use as a carrier in aerosols was abandoned in the United States in 1973. Despite widespread abuse $^{3}$ interest has focused on sudden deaths after acute overdosage. Chronic cardiac damage has not been recognised as a hazard of repeated exposure. There is a chemical similarity to halothane (both agents are halogenated $2 \mathrm{C}$ hydrocarbons) but no prior evidence of interaction has been reported. We postulate that such an interaction may partly explain the cardiac deterioration in our two patients. These cases also illustrate that the cardiac damage sustained after exposure to trichloroethane may be severe and life threatening and may be irreversible.

We thank Dr E G J Olsen, of the National Heart Hospital, London, for the histological report in case 2 .

\section{References}

1 Health and Safety Executive. Toxicity review 9: 1,1,1-trichloroethane. London: HMSO, 1984

2 Feigenbaum H. Echocardiography. 3rd ed. Philadelphia: Lea and Febiger, 1981:550.

3 Thomas Y. Kicking the glue buzz. Sunday Times 1986;Oct 12:52.

4 Wise MG, Fisher JG, de la Pena AM. Trichloroethane (TCE) and central sleep apnea: a case study. f Toxicol Environ Health 1983;11:101-4.

5 Wright MF, Strobl DJ. 1,1,1-Trichloroethane cardiac toxicity: report of a case. $\mathcal{F}$ Am Osteopath Assoc 1984;84:285-8.

6 Marshall BE, Wollman H. General anesthetics. In: Gilman AG, Goodman LS, Rall TW, Murad F, eds. The pharmacological basis of therapeutics 7th ed. London: Macmillan, 1985:279.

(Accepted 23 January 1987)

\title{
Digoxin-like immunoreactive substance in patients with aneurysmal subarachnoid haemorrhage
}

\author{
E F M WIJDICKS, M VERMEULEN, P van BRUMMELEN, N C DEN BOER, J van GIJN
}

\begin{abstract}
Digoxin was measured by radioimmunoassay in the plasma of 25 patients with aneurysmal subarachnoid haemorrhage who had not received digoxin treatment. After heating the plasma an
\end{abstract}

\footnotetext{
Department of Neurology, University Hospital Dijkzigt, Rotterdam, The Netherlands

E F M WIJDICKS, MD, neurologist

$M$ VERMEULEN, MD, neurologist

Department of Nephrology, University Hospital, Leyden
P VAN BRUMMELEN, MD, nephrologist

Department of Clinical Chemistry, University Hospital (Sophia Children's Hospital), Rotterdam

N C DEN BOER, MSC, clinical biochemist

Department of Neurology, University Hospital, Utrecht J VAN GIJN, MD, professor of neurology

Correspondence to: Dr E F M Wijdicks, Department of Neurology, University Hospital of Utrecht, 3511 HG Utrecht, The Netherlands.
}

endogenous substance cross reacting with antibodies to digoxin was identified in 18 cases. The presence of this substance was significantly related to the total amount of blood and to the presence of blood in the frontal interhemispheric fissure and could not be explained by hypertension or intake of water and sodium. A negative sodium balance and volume depletion occurred more often in patients who were positive for digoxin, but this relation did not reach statistical significance.

It is concluded that a digoxin-like natriuretic factor is released in response to a subarachnoid haemorrhage, probably as a result of hypothalamic damage.

\section{Introduction}

Hyponatraemia may occur after aneurysmal subarachnoid haemorrhage. ${ }^{1}$ In a consecutive series of 134 patients 44 were noted to have hyponatraemia during the first two weeks after the initial haemorrhage. ${ }^{2}$ None of these patients suffered neurological deterioration directly attributable to the hyponatraemia, probably because sodium concentrations fell gradually and not below $120 \mathrm{mmol} / \mathrm{l}^{3}$ Nevertheless, patients with hyponatraemia had a 